\title{
Sequence-specific modification of genomic DNA by small DNA fragments
}

\author{
Dieter C. Gruenert, ${ }^{1}$ Emanuela Bruscia, ${ }^{1,2}$ Giuseppe Novelli, ${ }^{2}$ Alessia Colosimo, 3,4 \\ Bruno Dallapiccola, ${ }^{3,5}$ Federica Sangiuolo, ${ }^{2}$ and Kaarin K. Goncz ${ }^{1}$ \\ ${ }^{1}$ Human Molecular Genetics Unit, Department of Medicine, University of Vermont, Burlington, Vermont, USA \\ 2Department of Biopathology and Diagnostic Imaging, University “Tor Vergata," Rome, Italy \\ ${ }^{3}$ CSS Mendel Institute, Rome, Italy \\ ${ }^{4}$ Department of Biomedical Sciences, University “G. D’Annunzio," Chieti, Italy \\ ${ }^{5}$ Department of Experimental Medicine and Pathology, University "La Sapienza," Rome, Italy
}

Small DNA fragments have been used to modify endogenous genomic DNA in both human and mouse cells. This strategy for sequence-specific modification or genomic editing, known as small-fragment homologous replacement (SFHR), has yet to be characterized in terms of its underlying mechanisms. Genotypic and phenotypic analyses following SFHR have shown specific modification of disease-causing genetic loci associated with cystic fibrosis, $\beta$-thalassemia, and Duchenne muscular dystrophy, suggesting that SFHR has potential as a therapeutic modality for the treatment of monogenic inherited disease.

J. Clin. Invest. 112:637-641 (2003). doi:10.1172/JCI200319773.

Gene therapy holds a great deal of promise for the future of medical treatment. However, success has been limited, partly because the field is still in its infancy ( 1 , 2). While initial prospects for cDNA-based gene therapy appeared quite promising, the anticipated breakthroughs did not materialize (3). The optimistic projections of success were, somewhat naively, based primarily on in vitro studies and/or model systems that had a limited relationship to the target organs. These projections underestimated the complexity of an intact biological system and the importance of genomic integrity in the cell-specific regulation of gene expression. As a result, a "best guess" approach to gene therapy has developed into an empirical, observation-based approach that is systematically building a foundation of knowledge on which clinical gene therapy will ultimately be based. This investigative foundation building has resulted in the establishment of numerous reagents and strategies intended to make gene therapy both safe and efficacious.

\footnotetext{
Address correspondence to: Dieter C. Gruenert, California Pacific Medical Center Research Institute, Stern Building, 2330 Clay Street, San Francisco, California 94115, USA Phone: (415) 600-1728; Fax: (415) 600-1390;

E-mail: dieter@cooper.cpmc.org.

Conflict of interest: The authors have declared that no conflict of interest exists.

Nonstandard abbreviations used: small DNA fragment (SDF); small-fragment homologous replacement (SFHR); singlestranded DNA (ssDNA); double-stranded DNA (dsDNA); zeocin resistance $\left(\mathrm{ZeO}^{\mathrm{r}}\right)$; polyethylenimine $(\mathrm{PEI})$; cystic fibrosis transmembrane conductance regulator (CFTR); sickle globin ( $\beta^{\text {s-globin}) ; ~ h e m a t o p o i e t i c ~ p r o g e n i t o r ~ c e l l ~(H P C) . ~}$
}

In general, gene therapy has very straightforward goals: to correct the pathogenic phenotype that results from genetic mutation(s), and to ensure that the therapeutic strategy is safe for the patient (see Goals of gene therapy). Most current gene therapy approaches are based on the successful introduction and expression of cDNA in cells in vitro $(4,5)$. These cDNA-based approaches have demonstrated a degree of phenotypic correction; nevertheless, until recently, long-term efficacy in human clinical trials has been elusive.

Two recent studies have provided a glimmer of what the future may hold for cDNA-based gene therapy $(6,7)$. These studies, as well as previous cDNA-based studies, involved the introduction of WT cDNA that complements the genetic mutation that manifests the disease pathology. One study involved ex vivo treatment of hematopoietic stem/progenitor cells from patients with $\mathrm{X}$ chromosome-linked SCID with a recombinant retrovirus containing WT $\gamma$ c cytokine receptor cDNA. Patients were given autologous transplants of genetically modified hematopoietic stem/progenitor cells and now appear to mount a normal immune response to environmental stimuli (6). In another clinical trial, patients with factor VIII deficiency (which results in hemophilia A) were transplanted with autologous skin fibroblasts that had been transfected with a plasmid containing WT factor VIII cDNA (7). Unfortunately, one patient of the SCID clinical trial developed a $\mathrm{T}$ cell leukemia that appears to be linked to an insertional mutagenesis event resulting from the integration of the transgene (8).

As alternatives to the cDNA-based approaches to gene therapy, strategies that target the mutant region 
of the endogenous gene have been developed. These genome-based approaches circumvent some of the issues (e.g., insertional mutagenesis) that have been associated with viral cDNA-based methods by relying on nonviral DNA delivery vehicles. Gene-targeting strategies also maintain the integrity of the target gene in terms of the relationship between the protein coding sequences and the gene-specific regulatory elements. With the exception of an adeno-associated virus-based approach (9-11), most gene-targeting strategies are oligonucleotide based (12-18). The oligonucleotidebased gene-targeting strategies include those that use triplex-forming oligonucleotides (19-22), RNA/DNA hybrid oligonucleotides (23-26), and small DNA fragments (SDFs) (27-37). This Perspective will focus on one of these oligonucleotide strategies: small-fragment homologous replacement (SFHR) $(12,13,32,36)$. The SDFs used in SFHR are composed of either singlestranded DNA (ssDNA) or double-stranded DNA (dsDNA), contain noncoding sequences, and are essentially homologous to target loci. These SDFs effect homologous exchange between incoming SDF sequences and endogenous (genomic or episomal) sequences, ultimately resulting in phenotypic changes.

\section{SFHR-mediated gene targeting}

The SFHR gene-targeting strategy uses ssDNA and dsDNA fragments that are effectively homologous to genomic or episomal cDNA target sequences in order to catalyze intracellular enzymatic mechanisms that mediate homologous exchanges (12) (Figure 1). SFHR has been applied in human epithelial and hematopoietic cells as well as mouse hematopoietic and ES cells (refs. 29, 32, 36, 38; and G. Novelli et al., unpublished observations). As many as 4 bp's have been concomitantly inserted, deleted, and/or altered by SFHR, and to date, studies suggest a broad range of utility in terms of target genes and cell types able to support SFHR. Moreover, SFHR appears to be effective both in vitro and in vivo.

\section{Model systems}

A group of independent studies in human airway epithelial cells was used to quantitatively evaluate SFHR and the effectiveness of various DNA delivery systems $(39,40)$. These studies were focused on the correction of a 4-bp insertion mutation that inactivated the zeocin resistance $\left(\mathrm{Zeo}^{\mathrm{r}}\right)$ gene carried in both a prokaryotic and a eukaryotic expression vector (31). This insertion mutation both inactivated the Zeor gene and eliminated an XmaI restriction enzyme cleavage site.
Upon correction, $\mathrm{Zeo}^{\mathrm{r}}$ and XmaI cleavage were restored. Using a transient transfection assay in which the vector containing the mutant $\mathrm{Zeo}^{\mathrm{r}}$ gene and SDFs comprising WT Zeor sequences were cotransfected, up to $4 \%$ of the vector recovered from the transfected human cells gave rise to $\mathrm{Zeo}^{\mathrm{r}}$ bacteria. Functional assays, restriction digests, and sequence analysis demonstrated that these Zeo $^{\mathrm{r}}$ colonies contained plasmids with an SFHR-corrected WT Zeor gene. In addition, these studies indicated that cationic DNA transfer vehicles (liposomes and polyamidoamine dendrimers) can sequester the target DNA and/or the SDF such that it is not readily accessible to the enzymatic machinery that facilitates SFHRmediated exchange. This was evident from the observation that the frequency of SFHR-mediated exchange was two orders of magnitude higher when bacteria were transformed with plasmids derived from nuclear extracts, as compared with plasmids derived from wholecell extracts. This is in sharp contrast with studies comparing whole-cell and nuclear plasmid extracts from electroporated epithelial cells, in which no difference in SFHR-mediated modification frequency between the two extraction procedures was observed (31).

Another recent study, using a defective GFP reporter plasmid as the target DNA, allowed real-time measurement of SFHR-mediated functional correction of an episomal target plasmid in live mammalian cells (40). SDFs (442 bp in length) were mixed with polyethylenimine (PEI) and delivered as a complex with a mutant GFP plasmid to variety of different cell lines. Functional cor-
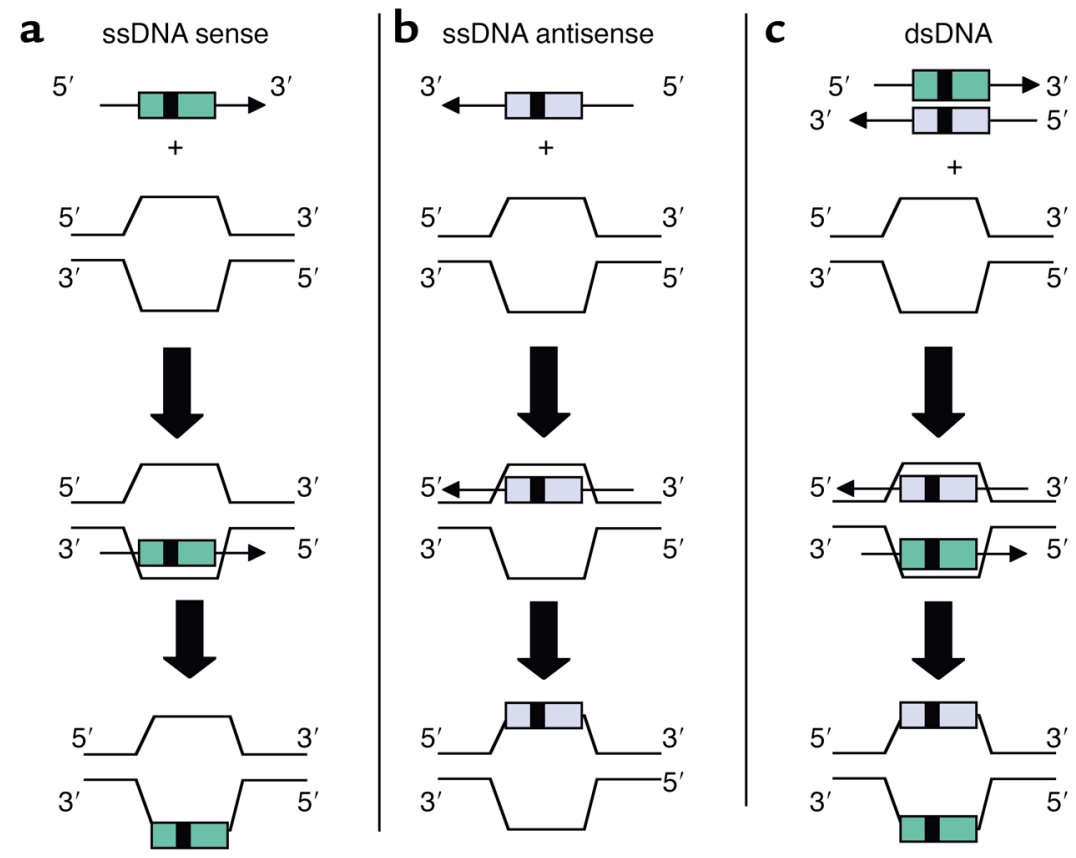

Figure 1

Schematic representation of different SDF configurations possible in SFHR-mediated modification of a target sequence. The SDF can interact with the target sequence as (a) sense and/or (b) antisense ssDNA, or as (c) dsDNA. The ssDNA can be introduced either as individual strands (sense and antisense) or as denatured cDNA strands. The enzymatic mechanisms involved require further elucidation. 


\begin{tabular}{|c|c|}
\hline \multicolumn{2}{|c|}{ Goals of gene therapy } \\
\hline \multicolumn{2}{|c|}{ 1. Correction of } \\
\hline 2. & Expression of normal protein and phenotype \\
\hline 3. & $\begin{array}{l}\text { Long-term correction with minimal application or multiple } \\
\text { applications with nontoxic or nonimmunogenic methods } \\
\text { of delivery }\end{array}$ \\
\hline 4. & Rapid and efficient delivery \\
\hline
\end{tabular}

rection of the mutant reporter gene was phenotypically assessed as a restoration of green fluorescence and was measured by FACS analysis of individual cells. Targeted correction efficiencies ranging from $0.1 \%$ to $1.2 \%$ were observed in five different mammalian cell lines.

In a follow-up study, stable cell lines carrying an integrated GFP gene were created to assess the efficiency of gene conversion (40). Larger SDFs (up to 1,600 bp in length) were more efficient in mediating the homologous exchange of the endogenous mutant GFP gene. However, the efficiency observed in genomic targeting was 100 -fold lower than that observed with episomal correction. This may, in part, be due to the delivery of SDFs into the nuclei of target cells, which was observed to be relatively inefficient when PEI-mediated transfection was used.

Model systems such as the Zeor and the GFP systems will be extremely useful for defining those parameters critical for optimization of SFHR, as well as for evaluating the effectiveness of cationic systems for the nuclear delivery of DNA. Clearly, extrapolation to other reporter or selectable marker gene systems can have important implications for high-throughput screening for effective DNA delivery systems.

\section{Cystic fibrosis}

Over the last decade, numerous in vitro SFHR studies were carried out in human airway epithelial cells, demonstrating both genotypic and phenotypic SFHRmediated modification of the cystic fibrosis transmembrane conductance regulator (CFTR) gene $(12,14$, $27,29,32,37,41,42)$. SDFs were applied in cultured human airway epithelial cells for SFHR-mediated correction of the CFTR gene by direct replacement of the $\Delta$ F508 mutation, a 3-bp deletion, with WT sequences $(27,29,32,37,41)$. Both genotypic and phenotypic measurements (e.g., chloride ion transport) indicated that SFHR-mediated modification occurred in 1-10\% of the transfected cells. The studies indicated not only that SFHR could directly modify genomic DNA, but also that multiple bases could be effectively altered at the same time. Moreover, these investigations clearly show that SFHR can facilitate multiple base insertions and deletions, as well as concomitant base transversions in genomic DNA. These in vitro studies resulted in restoration of the WT ion-transport phenotype.

Variations in SFHR-mediated gene-targeting efficiency can also be correlated with cell type and lipid/DNA charge ratio $(37,43)$. These factors appear to mediate both the internalization of SDF/liposome complexes and their intracellular fate. Previous observations have suggested that an additional element - plasma-membrane lipid composition, a function of the cell type - may define the lipid/DNA charge ratio (43). It also appears that this lipid/DNA charge ratio regulates the mechanism of lipoplex entry into the cells; i.e., lipid-DNA entry into the cells by endocytosis or membrane fusion depends on lipoplex charge ratio. Entry via an endocytic mechanism seems to be essential for the release of the DNA from the lipoplexes and for transport into the nucleus $(37,44)$. This apparent relationship between endocytic entry and nuclear delivery will likely have important implications for the delivery of any DNA.

Recent studies have also shown that such gene targeting can occur in mouse airway epithelial cells in vivo (28). The data indicate that SFHR-mediated modification occurs in mouse airways and intestine in cells that express mouse CFTR mRNA following transfection with various cationic lipid and dendrimer DNA complexes. These in vivo studies represent the first in a series of preclinical investigations necessary for quantification of SFHR modification and evaluation of its functional efficacy. In addition, these studies support previous in vitro observations showing that 4 bp's can be concomitantly modified even when one bp is distal from the primary mutation.

\section{Sickle cell anemia}

In another series of studies, the human $\beta$-globin gene was the target for SFHR (36). The aim of this work was modification of the $\beta$-globin gene, $H B B$, at codon 6 , the site of the mutation responsible for sickle cell anemia. These proof-of-principle studies demonstrated that SFHR-mediated conversion, from A to T at codon 6 , could be achieved by transfection of hematopoietic cells with $\beta$-globin SDFs comprising the sickle globin ( $\beta^{\text {s-globin}}$ ) mutation. The hematopoietic cells were either cell lines - i.e., murine erythroleukemia cells carrying human chromosome 11 (known as A181ß) or the human erythroleukemia cell line K562 - or human primitive hematopoietic

Genome-based oligonucleotide gene therapy

\section{Advantages}

Maintains gene integrity

Maintains relationship of coding and regulatory sequences

Retains cell-specific expression

Ensures appropriate level and duration of expression

Should be permanent

\section{Disadvantages}

May have low frequency

May result in random integration

May stimulate an apoptotic cascade

Degradation of therapeutic DNA may occur 
progenitor cell (HPCs). The A181 $\beta$ and K562 cell lines were transfected by electroporation, while the HPCs were transfected by microinjection. These two physical methods of transfection might overcome the inefficient nuclear delivery of DNA fragments that was observed in the model systems described above, in which chemical delivery vehicles were used. Besides demonstrating SFHR-mediated modification of the $\beta$-globin gene with SDFs, the studies showed that the $\beta$-globin gene in human airway epithelial cells can

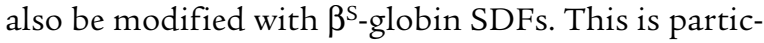
ularly noteworthy in that these cells do not express the $\beta$-globin gene, implying that transcription is not necessary for SFHR-mediated modification.

The studies in HPCs showed that SFHR-mediated conversion of WT $\beta$-globin (known as $\beta^{\mathrm{A}}$-globin) to the sickle cell $\beta^{\mathrm{S}}$-globin was stable in a culture that was expanded from $10^{3}$ to $10^{5}$ cells (36). These studies also indicated that, at a minimum, $1-2 \%$ of the cells had been modified by SFHR. This was determined from the fact that 60 of 100 injected cells were viable in the starting population of $10^{3}$ cells. Thus, if only one in 60 cells underwent SFHR-mediated conversion, a minimum of $1-2 \%$ of the cells would have been converted.

\section{Duchenne muscular dystrophy}

Another target for SFHR-mediated modification has been the Duchenne muscular dystrophy (DMD) analogue in the $m d x$ mouse model of DMD (30). In vitro and in vivo application of a WT SDF (603 bp) was used to facilitate a $\mathrm{T} \rightarrow \mathrm{C}$ WT conversion of a $\mathrm{C} \rightarrow \mathrm{T}$ nonsense mutation in $m d x$ exon 23 of the dystrophin gene, dys. Multiple applications of the WT SDF and variations in the Lipofectamine transfection complex enhanced the efficiency of SFHR-mediated modification in vitro. Conversion was observed at both the DNA and the RNA levels when the cells and tissue were analyzed by PCR or RT-PCR amplification, respectively. The efficiency of conversion of the $m d x$ mutant locus to the WT dys was about $15-20 \%$ in vitro and up to $0.1 \%$ in the tibialis anterior in vivo. Correction in myoblasts from $m d x$ mice persisted at least 28 days in culture and up to 3 weeks in vivo. These studies are very encouraging for the potential development of SFHR-based therapies for neuromuscular disorders.

\section{Summary}

The ability to correct a mutation in genomic DNA is clearly a desirable goal for gene therapy. There are advantages and limitations to this approach (see Genome-based oligonucleotide gene therapy). The advantages include the ability to maintain gene integrity and the relationship between the protein coding sequences and the gene-regulatory elements. This would overcome any potential for inappropriate gene expression either in the amount of protein produced or in the cell type expressing the gene. The limitations to a genomic correction/modification approach include the possibility that the modification would not be efficient enough to pro- duce a viable therapeutic outcome. Previous homologous-recombination studies in mouse ES cells, using large bacterial plasmids to carry large homologous segments of mouse DNA into the ES cells, indicated that the efficiencies of homologous recombination were low $\left(\leq 10^{-5}\right)(45)$. While these studies were elegant, they were based on the introduction of both homologous (in the form of genomic DNA segments) and nonhomologous (bacterial plasmid and selectable marker gene) dsDNA sequences into ES cells. The homologous regions were often interspersed with nonhomologous sequences and therefore would be thermodynamically less stable in homologous pairing than would pieces of DNA that were effectively homologous to the target genomic DNA. One early study, in which small pieces of dsDNA were injected into ES cells, demonstrated that 1 in 150 cells underwent homologous exchange when the DNA segments were effectively at least $99 \%$ homologous to the target genomic DNA (46), although these high frequencies were not always observed in other cell systems using a variety of DNA delivery vehicles $(33,35)$.

More recently, a number of different oligonucleotide strategies have reported frequencies similar to those indicated following the microinjection of ES cells $(12-15,17,18)$. These studies provide evidence to suggest that such strategies might be viable alternatives to cDNA-based gene therapy systems now being evaluated in clinical trials. The SFHR strategy outlined in this overview has demonstrated utility for sequence-specific genome editing of three endogenous genes: CFTR, $H B B$, and dys. SFHR has demonstrated the capacity to elicit single-base substitutions as well as concomitant insertion or deletion of multiple bases at efficiencies that could approach therapeutic viability.

A critical step in the development of SFHR as a therapeutic intervention will be the elucidation of the molecular mechanism(s) that underlies the homologous replacement observed after the introduction of SDFs. Dissection of the molecular basis of SFHR will be greatly facilitated by assessment of SFHR-mediated modification in cell-free extracts, bacteria, or eukaryotic cells defective in DNA repair, replication, or recombination pathways, coupled with microarray analysis of changes in gene expression after the introduction of SDFs.

SFHR, like other gene-editing strategies, has the potential both to be applied therapeutically and to enhance the understanding of disease through the development of transgenic animal models of disease. Advances in bone marrow-derived stem cell technology $(15,47-53)$ have suggested opportunities for expanding the range of genetic diseases that can be effectively treated by ex vivo gene targeting in pluripotent stem cells. Preliminary studies in mouse ES cells and in bone marrow-derived stem cells indicate that these cells can be genetically modified by SFHR (G. Novelli et al., unpublished observations). These studies on human cells have significant implications for the potential of using SFHR-modified stem cells for tissue repair in organs damaged by genetic disease-related pathology. 


\section{Acknowledgments}

The studies presented were supported by grants to Dieter C. Gruenert, Kaarin K. Goncz, and Emanuela Bruscia from the Cystic Fibrosis Foundation and Pennsylvania Cystic Fibrosis Research Inc. Giuseppe Novelli, Federica Sangiuolo, and Alessia Colosimo were supported by grants from the Italian Ministry of Health and the Italian Ministry of University and Scientific Research. Emanuela Bruscia is also a fellow of the Associazione Studio Atrofia Muscolare Spinale Infantile (ASAMSI), Italy. Bruno Dallapiccola received support from the Italian Ministry of Health and San Giovanni Rotondo. We would like to thank Valentina Guida for providing Figure 1.

1. Friedmann, T. 1989. Progress toward human gene therapy. Science. 244:1275-1281.

2. Friedmann, T. 1992. A brief history of gene therapy. Nat. Genet. 2:93-98. 3. Marshall, E. 1995. Gene therapy's growing pains. Science. 269:1050-1055.

4.2001. Summary table: human gene therapy protocols, last updated 8/10/01. Hum. Gene Ther. 12:2019-2020.

5. Gene therapy clinical trials. The Journal of Gene Medicine. http:// www.wiley.co.uk/genetherapy/clinical/.

6. Cavazzana-Calvo, M., et al. 2000. Gene therapy of human severe combined immunodeficiency (SCID)-X1 disease. Science. 288:669-672.

7. Roth, D.A., Tawa, N.E., Jr., O’Brien, J.M., Treco, D.A., and Selden, R.F. 2001. Nonviral transfer of the gene encoding coagulation factor VIII in patients with severe hemophilia A. N. Engl.J. Med. 344:1735-1742.

8. Marshall, E. 2002. What to do when clear success comes with an unclear risk. Science. 298:510-511.

9. Inoue, N., Dong, R., Hirata, R.K., and Russell, D.W. 2001. Introduction of single base substitutions at homologous chromosomal sequences by adeno-associated virus vectors. Mol. Ther. 3:526-530.

10. Russell, D.W., and Hirata, R.K. 1998. Human gene targeting by viral vectors. Nat. Genet. 18:325-330.

11. Russell, D.W., Hirata, R.K., and Inoue, N. 2002. Validation of AAV-mediated gene targeting. Nat. Biotechnol. 20:658.

12. Gruenert, D.C. 1998. Gene correction with small DNA fragments. Current Research in Molecular Therapeutics. 1:607-613.

13. Gruenert, D.C. 1999. Opportunities and challenges in targeting genes for therapy. Gene Ther. 6:1347-1348.

14. Lai, L.W., and Lien, Y.H. 1999. Homologous recombination based gene therapy. Exp. Nephrol. 7:11-14.

15. Richardson, P.D., Augustin, L.B., Kren, B.T., and Steer, C.J. 2002. Gene repair and transposon-mediated gene therapy. Stem Cells. 20:105-118.

16. Richardson, P.D., Kren, B.T., and Steer, C.J. 2001. Targeted gene correction strategies. Curr. Opin. Mol. Ther. 3:327-337.

17. Vasquez, K.M., Marburger, K., Intody, Z., and Wilson, J.H. 2001. Manipulating the mammalian genome by homologous recombination. Proc. Natl. Acad. Sci. U. S. A. 98:8403-8410.

18. Yanez, R.J., and Porter, A.C. 1998. Therapeutic gene targeting. Gene Ther. 5:149-159.

19. Vasquez, K.M., Narayanan, L., and Glazer, P.M. 2000. Specific mutations induced by triplex-forming oligonucleotides in mice. Science. 290:530-533.

20. Vasquez, K.M., and Wilson, J.H. 1998. Triplex-directed modification of genes and gene activity. Trends Biochem. Sci. 23:4-9.

21. Datta, H.J., Chan, P.P., Vasquez, K.M., Gupta, R.C., and Glazer, P.M 2001. Triplex-induced recombination in human cell-free extracts. Dependence on XPA and HsRad51. J. Biol. Chem. 276:18018-18023.

22. Vasquez, K.M., Dagle, J.M., Weeks, D.L., and Glazer, P.M. 2001. Chromosome targeting at short polypurine sites by cationic triplex-forming oligonucleotides. J. Biol. Chem. 276:38536-38541.

23. Wu, X.S., Liu, D.P., and Liang, C.C. 2001. Prospects of chimeric RNADNA oligonucleotides in gene therapy. J. Biomed. Sci. 8:439-445.

24. Kren, B.T., Metz, R., Kumar, R., and Steer, C.J. 1999. Gene repair using chimeric RNA/DNA oligonucleotides. Semin. Liver Dis. 19:93-104.

25. Yoon, K., Cole-Strauss, A., and Kmiec, E.B. 1996. Targeted gene correc- tion of episomal DNA in mammalian cells mediated by a chimeric RNADNA oligonucleotide. Proc. Natl. Acad. Sci. U. S. A. 93:2071-2076.

26. Alexeev, V., and Yoon, K. 1998. Stable and inheritable changes in genotype and phenotype of albino melanocytes induced by an RNA-DNA oligonucleotide. Nat. Biotechnol. 16:1343-1346.

27. Goncz, K.K., and Gruenert, D.C. 2000. Site-directed alteration of genomic DNA by small-fragment homologous replacement. Methods Mol. Biol. 133:85-99.

28. Goncz, K.K., et al. 2001. Expression of DeltaF508 CFTR in normal mouse lung after site-specific modification of CFTR sequences by SFHR. Gene Ther. 8:961-965.

29. Goncz, K.K., Kunzelmann, K., Xu, Z., and Gruenert, D.C. 1998. Targeted replacement of normal and mutant CFTR sequences in human airway epithelial cells using DNA fragments. Hum. Mol. Genet. 7:1913-1919.

30. Kapsa, R., et al. 2001. In vivo and in vitro correction of the mdx dystrophin gene nonsense mutation by short-fragment homologous replacement. Hum. Gene Ther. 12:629-642.

31. Colosimo, A., Goncz, K.K., Novelli, G., Dallapiccola, B., and Gruenert, D.C. 2001. Targeted correction of a defective selectable marker gene in human epithelial cells by small DNA fragments. Mol. Ther. 3:178-185.

32. Kunzelmann, K., et al. 1996. Gene targeting of CFTR DNA in CF epithelial cells. Gene Ther. 3:859-867.

33. Campbell, C.R., Keown, W., Lowe, L., Kirschling, D., and Kucherlapati, R. 1989. Homologous recombination involving small single-stranded oligonucleotides in human cells. New Biol. 1:223-227.

34. Gareis, M., Harrer, P., and Bertling, W.M. 1991. Homologous recombination of exogenous DNA fragments with genomic DNA in somatic cells of mice. Cell. Mol. Biol. 37:191-203.

35. Hunger-Bertling, K., Harrer, P., and Bertling, W. 1990. Short DNA fragments induce site specific recombination in mammalian cells. Mol. Cell. Biochem. 92:107-116.

36. Goncz, K.K., Prokopishyn, N.L., Chow, B.L., Davis, B.R., and Gruenert, D.C. 2002. Application of SFHR to gene therapy of monogenic disorders. Gene Ther. 9:691-694.

37. Sangiuolo, F., et al. 2002. In vitro correction of cystic fibrosis epithelial cell lines by small fragment homologous replacement (SFHR) technique. BMC Med. Genet. 3:8-19.

38. Gruenert, D.C., et al. 1998. Gene targeting in somatic cells: prospects for the future of gene therapy. Presented at: 6th Max Delbruck Symposium on Gene Therapy, May 6-8. Berlin, Germany. (Abstr.)

39. Colosimo, A., Guida, V., Palka, G., and Dallapiccola, B. 2002. Extrachromosomal genes: a powerful tool in gene targeting approaches. Gene Ther. 9:679-682.

40. Thorpe, P., Stevenson, B.J., and Porteous, D.J. 2002. Optimising gene repair strategies in cell culture. Gene Ther 9:700-732.

41. Bruscia, E., et al. 2002. Isolation of CF cell lines corrected at DeltaF508CFTR locus by SFHR-mediated targeting. Gene Ther. 9:683-685.

42. Goncz, K.K., and Gruenert, D.C. 2001. Modification of specific DNA sequences by small fragment homologous replacement. Biotechnology International III. 3:113-119.

43. Colosimo, A., et al. 2000. Transfer and expression of foreign genes in mammalian cells. Biotechniques. 29:314-331.

44. Holmes, A.R., Dohrman, A.F., Ellison, A.R., Goncz, K.K., and Gruenert, D.C. 1999. Intracellular compartmentalization of DNA fragments in cultured airway epithelial cells mediated by cationic lipids. Pharm. Res. 16:1020-1025.

45. Capecchi, M.R. 1994. Targeted gene replacement. Sci. Am. 270:52-59.

46. Zimmer, A., and Gruss, P. 1989. Production of chimaeric mice containing embryonic stem (ES) cells carrying a homoeobox Hox 1.1 allele mutated by homologous recombination. Nature. 338:150-153.

47. Abkowitz, J.L. 2002. Can human hematopoietic stem cells become skin, gut, or liver cells? N. Engl. J. Med. 2346:770-772.

48. Adam, D. 2002. Britain banks on embryonic stem cells to gain competitive edge. Nature. 416:3-4

49. Cibelli, J.B., et al. 2002. Parthenogenetic stem cells in nonhuman primates. Science. 295:819.

50. Jiang, Y., et al. 2002. Pluripotency of mesenchymal stem cells derived from adult marrow. Nature. 418:41-49.

51. Krause, D.S., et al. 2001. Multi-organ, multi-lineage engraftment by a single bone marrow-derived stem cell. Cell. 105:369-377.

52. Krause, D.S. 2002. Plasticity of marrow-derived stem cells. Gene Ther. 9:754-758.

53. Theise, N.D., and Krause, D.S. 2002. Toward a new paradigm of cell plasticity. Leukemia. 16:542-548. 